\title{
Correlation between Central and Lateral Neck Dissection in Differentiated Thyroid Carcinoma
}

\author{
Olivia Mazzaschi ${ }^{1}$, Marine Lefevre ${ }^{2}$, Bruno Angelard ${ }^{1}$, Nathalie Chabbert-Buffet ${ }^{3}$, \\ Jean Lacau St. Guily ${ }^{1}$, Sophie Périé ${ }^{1}$ \\ ${ }^{1}$ Department of Otolaryngology-Head and Neck Surgery, Faculty of Medicine, University Pierre et Marie Curie Paris VI, \\ Tenon Hospital, Assistance Publique-Hôpitaux de Paris, Paris, France \\ ${ }^{2}$ Department of Pathology, Faculty of Medicine, University Pierre et Marie Curie Paris VI, \\ Tenon Hospital, Assistance Publique-Hôpitaux de Paris, Paris, France \\ ${ }^{3}$ Department of Obstetrics, Gynaecology and Reproductive Medicine-Endocrinology Section, Faculty of Medicine, \\ University Pierre et Marie Curie Paris VI, Tenon Hospital, Assistance Publique-Hôpitaux de Paris, Paris, France \\ Email: sophie.perie@tnn.aphp.fr
}

Received June 18, 2012; revised July 25, 2012; accepted August 13, 2012

\begin{abstract}
Objective: To determine the histopathological correlation between central and lateral neck metastasis in differentiated thyroid carcinoma, and its potential therapeutic impact. Although the central neck dissection (CND) is recommended in differentiated thyroid carcinoma, the indication for lateral neck dissection (LND) remains controversial. Design: Retrospective study. Methods and Main Outcome Measures: Pathological analysis of systematic ipsilateral central neck dissection (CND) and LND performed with total thyroidectomy in differentiated thyroid carcinoma was retrospectively reviewed according to "side" and to "patient". Results: A total of 56 sides (46 patients) were suitable for analysis. Analysis by "side" revealed that CND and LND dissection samples were both negative in 15 cases, both positive in 32, CND was positive and LND was negative for 8 cases and CND was negative and LND was positive in 1 case. The combined presence of positive LND and positive CND was therefore observed in 32/40 "sides" and 26/46 "patients". Analysis by "side" of the impact of the treatment decision to perform ipsilateral LND only in patients with positive CND and vice versa demonstrated a sensitivity, specificity, and accuracy of $97 \%, 65 \%$, and $84 \%$, respectively. Conclusions: In most cases, the presence of positive LND was associated with positive ipsilateral CND. The very low prevalence of positive LND in patients with negative CND may justify LND as a second step procedure only in patients with positive CND, except in the case of documented lateral neck metastasis.
\end{abstract}

Keywords: Central Neck Dissection; Lateral Neck Dissection; Differentiated Thyroid Carcinoma; Pathological Analysis; Thyroid Carcinoma Neck Metastasis

\section{Introduction}

Discussion on the treatment of differentiated thyroid carcinoma currently focuses on the indications for prophylactic neck dissection. It has been shown that central neck dissection (CND) should be routinely combined with thyroidectomy in papillary carcinoma and microcarcinoma with aggressive criteria to decrease the risk of recurrence $[1,2]$. CND also allows accurate disease staging. Nevertheless, the higher incidence of recurrent laryngeal nerve palsy and hypoparathyroidism following re-operation has led to a consensus to perform bilateral CND (ipsilateral and contralateral paratracheal subsites and pretracheal and superior mediastinal subsites) at the time of primary surgery $[3,4]$.

However, the indication for lateral neck dissection (LND) remains controversial, especially in patients with no clinical and/or ultrasound evidence of lateral lymph node metastasis. In fact, there is commonly a discrepancy between the high frequency of pathological lymph node metastasis and the low rate of clinical or ultrasound lymph node involvement. Criteria of aggressive thyroid tumour such as size larger than $3 \mathrm{~cm}$, multifocal tumour, extracapsular spread, vascular invasion [5], and central node involvement, are currently established only at final pathological analysis, resulting in a difficult preoperative decision regarding appropriate neck dissection. LND could possibly be withheld in patients without metastasis in the central compartment, as neck metastases commonly start by involving the central nodes and subsequently spread to lateral nodes. However, "skip" metastases (positive nodes in the LND, and negative nodes in the CND) have been reported in the literature in $6 \%$ to $19.7 \%$ of papillary carcinomas [6-8] and $4.2 \%$ to $5.5 \%$ of 
micropapillary carcinoma [9]. This rate may also be underestimated, as it includes patients undergoing therapeutic rather than prophylactic LND $[7,8,10,11]$. Various types of neck dissection were performed in previous series [11] and, in the study by Machens [8], the mediastinal subsite was included in the LND and the criteria for lateral lymph node clearance were not well defined [8].

When LND is indicated, neck dissection of at least levels II, III and IV [12] is required, as metastatic disease may involve all of these levels [7]. However, this requires large incisions with cosmetic sequelae in young patients and functional complications (spinal injury with shoulder weakness, chronic neck pain, chyle leakage $[5,11,13]$. For these reasons, the value of systematic LND may be questioned. Moreover, cervical lymph node metastasis does not have a major impact on survival [11].

The aim of this work was to study the correlation between histopathological results of CND and LND performed systematically during this period in our institution, in patients with differentiated thyroid carcinoma. Results of systematic CND and LND assessed according to "side" and according to "patient" were analyzed. The objective was to evaluate whether negative CND could be predictive of negative LND, in which case the strategy of a two-stage surgical management would be validated. A first-step CND only procedure would limit the cosmetic and functional sequels. Subsequent LND would then be performed only in the case of positive CND.

\section{Patients and Methods}

\subsection{Patients}

Forty seven consecutive patients (11 males, 36 females: mean age: 46.82 years; range: 19 to 82 years) treated for differentiated thyroid carcinoma were included in this retrospective study from August 2000 to October 2008 in the Department of Otolaryngology Head and Neck Surgery, at Tenon Hospital, Paris. All patients underwent preoperative thyroid and neck ultrasonography. Distant metastasis was already present at the time of surgery in one patient.

The inclusion criteria were patients surgically treated for papillary or follicular thyroid cancer, regardless of tumour size, by total thyroidectomy or completion thyroidectomy, with systematic CND associated with LND on at least one side (routine LND); patients without cervical lymph node metastasis detected preoperatively by physical examination or ultrasonography were therefore included. Patients undergoing CND only or LND only were excluded.

Diagnosis of differentiated thyroid carcinoma was suspected preoperatively by fine-needle aspiration cytology, intraoperatively on frozen section or at definitive pathological analysis. Surgery was performed in one or several steps, as the diagnosis of cancer can only be confirmed on definitive histopathological examination and some patients had undergone incomplete thyroidectomy or incomplete node dissection elsewhere.

\subsection{Surgical Strategy}

Surgery was performed by 4 experienced thyroid surgeons of our Department of Otolaryngology.

Total thyroidectomy or completion thyroidectomy was performed prior to CND and LND. CND and LND specimens were analyzed according to the Robbins standardized neck dissection terminology (2002) [12].

CND was performed from the body of the hyoid bone superiorly, the carotid sheath laterally and suprasternal notch inferiorly (level VI). This level was divided into 3 subsites during surgery: ipsilateral and contralateral paratracheal subsites and the laryngotracheomediastinal subsite including the prelaryngotracheal and superior mediastinal regions. Ipsilateral or bilateral CND was performed in 13 and 34 patients, respectively (total of 81 CND).

LND consisted of a radical, modified radical or selective LND, removing levels IIb, IIa, III and IV [12]. Ipsilateral LND was performed in 31 patients, and bilateral LND was performed in 16 patients (total of 63 LND).

Thyroidectomy specimens and neck dissections were meticulously oriented by the surgeon, marked on a diagram and sent for histopathological examination.

\subsection{Histological Examination}

Histopathological examination was performed by a single pathologist. Thyroidectomy and all cervical lymph nodes of all levels of the neck dissections were fixed in formaldehyde and examined. Each cervical lymph node was totally sectioned every $3 \mathrm{~mm}$ and $5 \mu \mathrm{m}$ sections were mounted and stained by haematoxylin-eosin-saffron (HES) and then examined by light microscopy. Papillary carcinoma and cervical lymph node metastasis were diagnosed in the presence of typical nuclear atypia. Thyroglobulin antibody immunohistochemistry was sometimes performed on lymph nodes in the case of follicular carcinoma.

Histopathological findings on thyroidectomy were scored as pathological for the presence of primary thyroid tumour $(\mathrm{T}+)$. The differentiated thyroid carcinoma histological subtype, tumour size, number of foci, and the presence of extracapsular spread and vascular or lymphatic invasion were included in the report. The number of cervical lymph nodes examined was also recorded. Results were scored as pathological $\mathrm{N}+$ for lymph node involvement or $\mathrm{N}-$ for no involvement. The lymph node' capsule status was also reported.

Cases in which no nodes were identified on histopa- 
thological examination of the CND were excluded from the series when (one patient and one side in another patient were excluded).

These data allowed classification of patients according to the pTN classification.

\subsection{Data Analysis}

The results of pathological examination of resected lymph nodes were expressed according to "side". Correlations between histopathological results of both CND (including ipsilateral paratracheal and tracheomediastinal subsites) and ipsilateral LND were studied according to "side" (CND+, CND-, LND+, LND-), and according to "patient".

The tracheomediastinal subsite of the CND was interpreted as positive or negative together with the ipsilateral CND.

\subsection{Impact on Management}

The impact of neck dissection on the management of differentiated thyroid carcinoma was studied on the basis of the correlation between CND and LND pathology results. The hypothesis of a strategy consisting of performing systematic LND in patients with positive CND, and withholding LND in patients with negative CND was analysed. This strategy would require one- or two-stage surgery, depending on the results of pathological examination of the thyroidectomy and CND specimens. The sensitivity, specificity, accuracy, positive predictive value (PPV) and negative predictive value (NPV) were calculated.

\section{Results}

Twenty three patients underwent a one-stage surgical procedure comprising total thyroidectomy and simultaneous CND and LND. The other patients were operated in 2 or 3 stages, in the light of the final histopathological diagnosis (13 patients) or because the patient was re-

Table 1. Patients' characteristics.

\begin{tabular}{cc}
\hline Number of patients & 47 \\
\hline Men/women & $11 / 36$ \\
Age (years): mean (range) & 46.82 (19 to 82) \\
Time of surgery & \\
1 step & 23 \\
2 steps & 18 \\
3 steps & 6 \\
Thyroid tumor & \\
Papillary/vesicular/both & $42 / 2 / 3$ \\
Mean Size (mm) & $25.75(1$ to 60$)$ \\
Unifocal/Multifocal & $20 / 27$ \\
Unilateral/Bilateral & $28 / 19$ \\
Extracapsular spread & 21 \\
\hline
\end{tabular}

ferred to our department for neck dissection following thyroidectomy (11 patients) either for prophylaxis or for persistent disease. Patient characteristics are reported in Table 1.

\subsection{Histopathology of Thyroid Carcinoma}

This series comprised 42 papillary carcinomas, 2 follicular carcinomas and 3 patients presented both papillary carcinoma and follicular carcinoma. The tumour was unifocal in 20 patients and multifocal in 27 patients. The tumour was unilateral in 28 patients and bilateral in 19 patients (Table 1). Thyroid tumour was stage T1 in 13 cases, T2 in 7 cases, T3 in 17 cases and T4 in 10 cases.

\subsection{Histopathology of Lymph Node Dissections}

Since bilateral CND and LND were performed in 11 patients, a total of 58 dissection specimens were studied in 47 patients. However, 2 sides were excluded from the analysis, as the CND did not contain any nodes for histopathological examination: the side dissected in one male patient with a focal $\mathrm{T} 3$ papillary carcinoma with extracapsular spread (patient excluded) and one of the two sides dissected (one side excluded) in another patient with papillary carcinoma.

A total of 56 sides (56 CND and 56 LND on the same side) from 46 patients were finally studied to evaluate a correlation according to "side" and according to "patient".

Central neck metastasis was present in 40 CND (33 patients) and absent in 16 CND (13 patients). The number of lymph nodes included in the CND ranged from 1 to 10 nodes (mean: 3.34 nodes); the number of positive lymph nodes ranged from 1 to 5 (mean: 1.86). At least one lymph node presented signs of extracapsular spread in a total of 15 sides (12 patients).

Lateral neck metastasis was present in 33 LND (27 patients), and absent in 23 LND (19 patients). One patient was considered to be positive for the analysis according to patient, as one side was positive for both CND and LND and the other side was positive for CND and negative for LND. The number of lymph nodes included in the LND ranged from 5 to 69 nodes (mean: 23.36) and the number of positive lymph nodes ranged from 1 to 20 (mean: 5.14). At least one lymph node presented signs of extracapsular spread in 18 LND (16 patients).

Staging according to the pTNM classification for nodes (N0: no metastatic nodes, N1a: ipsilateral or upper mediastinal nodes, N1b contralateral or bilateral lymph nodes) showed 12 N0, 20 N1a and 14 N1b.

\subsection{Correlation between CND and LND According to "Side" and According to "Patient"}

Analysis according to "side" showed that CND and LND 
were both negative on 15 sides, CND and LND were both positive on 32 sides, CND was positive with LND was negative on 8 sides and CND was negative and LND was positive ("skip lateral metastasis") on 1 side (Tables 2 and 3). Consequently, in the presence of a positive CND, the LND was positive in 32 out of 40 "sides" $(80 \%)$; and 26 out of 46 patients $(56.52 \%)$. In contrast, a positive LND in the presence of a negative CND was observed in only 1 out of 16 "sides" $(6.25 \%)$ and only 1 out of 46 patients $(2.17 \%)$.

In the patient with a positive ipsilateral LND and negative CND ("skip" metastasis), the papillary carcinoma was diagnosed in a lateral cervical node.

A different lymph node metastasis status (positive bilateral CND, with positive LND on one side and negative LND on the other side) was demonstrated in only one of the patients undergoing bilateral CND and LND. Lymph node status was similar on both sides in all other patients.

In the two patients with follicular carcinoma, CND and LND were both positive in one case and CND and LND were both negative in the other case.

\subsection{Potential Impact on Management}

In patients with a positive CND, the decision to systematically perform ipsilateral LND would have resulted in 32 useful LND (26 patients) and 8 useless LND (7 patients).

In patients with a negative CND, the decision not to perform prophylactic ipsilateral LND would have missed one positive case ("skip" metastasis) (one patient). However, in this patient, papillary carcinoma was detected in a lateral cervical lymph node removed during neck dissection.

The sensitivity and specificity for this surgical strategy according to side were $96.96 \%(32 / 33)$ and $65.21 \%(15 /$ $23)$, respectively, with an accuracy of $83.92 \%$ (47/56), a PPV of $80 \%(32 / 40)$ and a NPV of $93.75 \%(15 / 16)$.

\subsection{Follow-Up}

All patients were followed until January 2012. The mean follow-up was 81.6 months (range: 42 to 137 ). Two patients, free of thyroid disease, died from an unrelated cause. Three patients exhibited cervical lymph node recurrence (1 in level V, 1 in the mediastinal area and the other in bilateral level III and IV); all of these patients were previously $\mathrm{N}+$ in both LND and CND. All of these

Table 2. Histopathological correlation between the 56 ipsilateral CND and LND at "side level".

\begin{tabular}{ccccc}
\hline CND & CND- & CND + & CND + & CND- \\
LND & LND- & LND + & LND - & LND + \\
\hline Total & 15 & 32 & 8 & 1 \\
\hline
\end{tabular}

CND: central neck dissection; LND: lateral neck dissection.
Table 3. Value of true positive, true negative, false positive and false negative cases at "side level".

\begin{tabular}{cccl}
\hline & LND - & LND & Total \\
\hline CND- & $15 \mathrm{TN}$ & $1 \mathrm{FN}$ & 16 \\
$\mathrm{CND}+$ & $8 \mathrm{FP}$ & $32 \mathrm{TP}$ & 40 \\
Total & 23 & 33 & 56 \\
\hline
\end{tabular}

CND: central neck dissection; LND: lateral neck dissection.

metastases were removed surgically followed by postoperative 131I therapy. One of these patients was considered to present progressive disease. A total of 44 patients were considered free of thyroid disease at present time.

\section{Discussion}

The treatment of differentiated thyroid carcinoma has not yet been standardized, especially in relation to lymph node dissection. This difficulty is partly due to the long survival and disease-free interval associated with these tumours, making it difficult to conduct prospective studies comprising identical treatment procedures. The maximalist or minimalist approach to treatment remains controversial. In addition, because of the diversity of surgeons treating thyroid carcinoma and the therapeutic potential of postoperative 131I therapy, management of neck dissection is highly dependent on the surgeon and surgical skills. However, systematic CND has started to be recommended in the treatment guidelines for differentiated thyroid cancer $[14,15]$. This consensus is based on the finding that CND may improve survival by reducing the risk of nodal recurrence [2,3], and allows accurate tumour staging. Permanent morbidity is not increased by CND [4], although significant morbidity has been reported by Roh et al, especially hypoparathyroidism [11]. Recurrence and re-operation in the central compartment is associated with a higher risk of vocal cord paralysis, justifying also bilateral CND [3,4]. The high frequency of multifocal cancer may also be a criterion to perform bilateral CND. For these reasons, at the present time in our department, all patients with suspected thyroid cancer on preoperative examinations are routinely treated by bilateral CND, including ipsilateral and contralateral paratracheal subsites and pretracheal and superior mediastinal subsites. The high rate of multifocal thyroid carcinoma $(57.44 \%)$ also supports this strategy.

The indication for LND in differentiated thyroid carcinoma is even more controversial and remains unclear, except in patients with preoperative evidence of lateral lymph node metastasis (clinically, on ultrasonography, or in patients with positive fine-needle aspiration cytology) $[2,4,7,8,11,13,16]$, as the significant morbidity of LND must be taken into account $[5,13]$. However, the sensitivity and negative predictive value of palpation or ultra- 
sonography to detect lateral node metastasis are too low to guide the optimal treatment strategy due to the high rate of false-negative results $[17,18]$. Levels IIa, IIb, III and IV should be removed since metastatic disease may involve all of these levels; levels I, Va and $\mathrm{Vb}$ are more rarely involved [11], although some authors reported significant metastatic disease in levels $\mathrm{V}$ and $\mathrm{I}[6,13,19]$. However, metastatic lateral lymph nodes are commonly located in level IV (41\% to $75.9 \%$ ), III (57\% to $72.2 \%$ ) and level II (52\% with involvement in IIa of $72.2 \%$ and in IIb of $16.7 \%$ ) [7,13]. Consequently, in addition to the cosmetic sequelae of the incision, especially in young women, spinal injury, shoulder weakness, fibrosis and sensory loss of the neck, chyle leakage, Horner syndrome, phrenic nerve palsy, may be difficult to manage [5,11, 13]. However, some authors advocate prophylactic LND due to the discrepancy between occult and clinical recurrent disease [20].

Sentinel node biopsy has also demonstrated promising results $[21,22]$ but studies based on larger cohorts are required $[23,24]$. The biopsy site may be difficult to determine in the case of multifocal carcinoma, a common situation (57.44\% of this series) and it is also important to adopt a homogeneous and optimal routine strategy whenever surgery is performed. It is not yet a widespread technique.

Some studies have compared the results of CND and LND status, but the indication for LND remains theraeutic $[7,8,10,16]$. The mediastinal subsite of the CND was included in the lateral compartment in the study by Machens [8], making it difficult to compare the results of this study with those of other studies. Nevertheless, Roh et al concluded that CND and LND are mandatory in papillary thyroid carcinoma, as lateral metastasis is associated with central node involvement [7] and the $9.6 \%$ "skip" metastasis rate is considered to be an epiphenomenon [7]. However, the decision to perform LND based on the CND pathology report has never been studied, although quantitative correlations between central and lateral neck lymph node metastases in papillary thyroid cancer were demonstrated by Machens et al. [25].

In order to more clearly define the indications for LND in differentiated thyroid carcinoma, we studied the correlation between LND and CND according to "side", performed routinely in our department in patients with differentiated thyroid carcinoma. A very low percentage of positive LND with negative CND was observed, while a high risk of lateral metastases was observed in the case of positive CND. The low percentage of "skip" metastasis $(2.17 \%$ of patients) compared to other studies (6 to $19.7 \%$ ) $[6,8,26,27]$ is probably due to the fact that LND was performed systematically, in contrast with other studies in which LND was only performed in the case of suspected lymph node involvement on preoperative ex- amination $[7,8,10]$. The only case of "skip" metastasis in the present series concerned a patient with lateral cervical lymph node that was already positive at fine-needle aspiration cytology for metastasis of papillary thyroid carcinoma revealing the cancer. For this patient, a therapeutic LND would have been systematically planned. This series also included cases of micropapillary thyroid carcinoma, as the inclusion criteria did not comprise tumour size, and the exact tumour size and its aggressive characteristics were defined by the pathological analysis. A small cancer may also be T3 in the presence of extracapsular spread. Tumour size can be proposed as a preoperative criterion to evaluate the risk of lateral metastasis. However, no difference was observed in the present series between patients with tumours larger than $3 \mathrm{~cm}$ and patients with tumours smaller than $3 \mathrm{~cm}(66.6 \%$ vs $74.19 \%$ respectively for positive CND, and $46.6 \%$ vs $64.51 \%$ respectively for positive LND) but no conclusion can be drawn from this small cohort (additional analysis). Some studies have also shown that tumours located in the superior parts of the thyroid gland are likely to metastasize to the lateral neck compartment via superior thyroid arteries [9,24]. This issue was not addressed in this study. It can be hypothesized that the present study comprised two populations of thyroid carcinoma: one with cervical lymph node metastasis and one without cervical lymph node metastasis. Patients in whom a therapeutic LND was reported in previous series corresponded to the population with cervical lymph node metastasis.

In differentiated thyroid carcinoma, we therefore recommend primary total thyroidectomy with CND in patients without preoperative lateral cervical lymph node, followed by LND in patients with positive CND on final pathological analysis (Figure 1). The proposed strategy requires routine bilateral CND containing lymph nodes in the dissection samples. Consequently, patients in whom no lymph node was detected in the CND were excluded

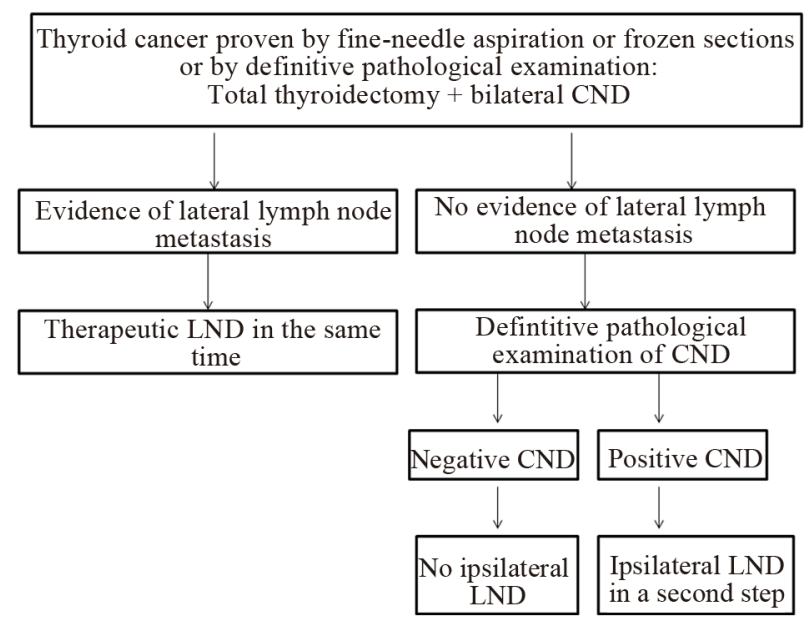

Figure 1. Operative strategy. 
from the analysis. The high rate of positive LND in the presence of positive CND (80\%) justifies this strategy although a percentage of these LND will be finally negative $(20 \%)$. The low rate of "skip" metastasis in the presence of negative CND also justifies this strategy. However, the possibility of "skip" metastases may constitute a limitation of this therapeutic approach. Nevertheless, postoperative 131I therapy can also be considered to be helpful in these cases in order to identify foci in the lateral neck compartment, allowing LND to be performed subsequently when serum thyroglobulin remains elevated. In 2004, Machens et al. also showed that "skip" metastases was "an epiphenomenon of low-intensity metastases and entails a moderate risk of local recurrence" [8].

In terms of patients, 38 out of 49 patients received adequate surgical treatment, surgery was excessive in 7 patients and was not sufficient in only 1 patient. An additional advantage of this strategy is that second-stage surgery, when required, can be performed in an appropriate department. Two-stage surgery can avoid unnecessary LND in patients with negative CND, with no prognostic impact for the other patients. This strategy reported for differentiated thyroid carcinoma remains to be studied in a larger prospective analysis.

\section{Conclusion}

The high prevalence of positive LND in patients with differentiated thyroid carcinoma and positive CND may justify subsequent LND based on the results of definitive histopathological examination of both the total thyroiddectomy and CND specimens. The low prevalence of "skip" metastasis in this study, in which CND and LND were performed systematically and studied according to side and according to patient, suggests that LND may be unnecessary in patients with negative CND. This study also suggests the existence of two populations of differentiated thyroid carcinoma, one with cervical lymph node metastasis and one without cervical lymph node metastasis.

\section{REFERENCES}

[1] M. L. White and G. M. Dohert, "Level VI Lymph Node Dissection for Papillary Thyroid Cancer," Minerva Chirurgica, Vol. 62, No. 5, 2007, pp. 383-393.

[2] S. H. Lee, S. S. Lee, S. M. Jin, J. H. Kim and Y. S. Rho, "Predictive Factors for Central Compartment Lymph Node Metastasis in Thyroid Papillary Microcarcinoma," Laryngoscope, Vol. 118, No. 4, 2008, pp. 659-662. doi:10.1097/MLG.0b013e318161f9d1

[3] M. L. White, P. G. Gauger and G. M. Doherty, "Central Lymph Node Dissection in Differentiated Thyroid Cancer 2007 by the Société Internationale de Chirurgie," World
Journal of Surgery, Vol. 31, No. 5, 2007, pp. 895-904. doi:10.1007/s00268-006-0907-6

[4] N. Palestini, A. Borasi, L. Cestino, M. Freddi, C. Odasso and A. Robecchi, "Is Central Neck Dissection a Safe Procedure in the Treatment of Papillary Thyroid Cancer? Our Experience," Langenbeck's Archives of Surgery, Vol. 393, No. 5, 2008, pp. 693-698. doi:10.1007/s00423-008-0360-0

[5] Y. Ito and A. Miyauchi, "Lateral Lymph Node Dissection Guided by Preoperative and Intraoperative Findings in Differentiated Thyroid Carcinoma," World Journal of Surgery, Vol. 32, No. 5, 2008, pp. 729-739. doi:10.1007/s00268-007-9315-9

[6] B. J. Lee, S. G. Wang, J. C. Lee, S. M. Son, I. J. Kim and C. I. Park, "Level IIb Lymph Node Metastasis in Neck Dissection for Papillary Thyroid Carcinoma," Archives of Otolaryngology-Head \& Neck Surgery, Vol. 133, No. 10, 2007, pp. 1028-1030. doi:10.1001/archotol.133.10.1028

[7] J. L. Roh, J. M. Kim and C. I. Park, "Lateral Cervical Lymph Node Metastases from Papillary Thyroid Carcinoma: Pattern of Nodal Metastases and Optimal Strategy for Neck Dissection," Annals of Surgical Oncology, Vol. 15, No. 4, 2008, pp. 1177-1182. doi:10.1245/s10434-008-9813-5

[8] A. Machens, H. J. Holzhausen and H. Dralle, "Skip Metastases in Thyroid Cancer. Leaping the Central Lymph Node Compartment," Archives of Surgery, Vol. 139, No. 1, 2004, pp. 43-45. doi:10.1001/archsurg.139.1.43

[9] N. Wada, Q. Y. Duh, K. Sugino, H. Iwasaki, K. Kameyna, T. Mimura, K. Ito, H. Takami and Y. Takanashi, "Lymph Node Metastasis from 259 Papillary Thyroid Microcarcinomas: Frequency, Pattern of Occurrence and Recurrence, and Optimal Strategy for Neck Dissection," Annals of Surgery, Vol. 237, No. 3, 2003, pp. 399-407. doi:10.1097/01.SLA.0000055273.58908.19

[10] A. Goropoulos, K. K. Karamoshos, A. Christodoulou, N. Theodoros, K. Paulou, A. Samaras, P. Xirou and I. Efstratiou, "Value of the Cervical Compartments in the Surgical Treatment of Papillary Thyroid Carcinoma," World Journal of Surgery, Vol. 28, No. 12, 2004, pp. 1275-1281. doi:10.1007/s00268-004-7643-6

[11] J. L. Roh, J. Y. Park and C. I. Park, "Total Thyroidectomy Plus Neck Dissection in Differentiated Papillary Thyroid Carcinoma Patients: Pattern of Nodal Metastasis, Morbidity, Recurrence, and Postoperative Levels of Serum Parathyroid Hormone," Annals of Surgery, Vol. 245, No. 4, 2007, pp. 604-610. doi:10.1097/01.sla.0000250451.59685.67

[12] T. Robbins, G. Clayman, P. A. Levine, J. Medina, R. Sessions, A. Shaha, P. Som and P. T. Wolf, "Neck Dissection Classification Update: Revisions Proposed by the American Head and Neck Society and the American Academy of Otolaryngology-Head and Neck Surgery," Archives of Otolaryngology-Head \& Neck Surgery, Vol. 128, No. 7, 2002, pp. 751-758.

[13] M. E. Kupferman, M. Patterson, S. J. Mandel, V. LiVolsi and R. S. Weber, "Patterns of Lateral Neck Metastasis in Papillary Thyroid Carcinoma," Archives of Otolaryngology-Head \& Neck Surgery, Vol. 130, No. 7, 2004, pp. 
857-860. doi:10.1001/archotol.130.7.857

[14] D. S. Cooper, G. M. Doherty, B. R. Haugen, R. T. Kloos, S. L. Lee, S. J. Mandel, E. L. Mazzaferri, B. McIver, S. I. Sherman and R. M. Tuttle, "Management Guidelines for Patients with Thyroid Nodules and Differentiated Thyroid Cancer. The American Thyroid Association Guidelines Taskforce," Thyroid, Vol. 16, No. 2, 2006, pp. 109-142. doi:10.1089/thy.2006.16.109

[15] F. Pacini, M. Schlumberger, H. Dralle, R. Elisei, J. W. A. Smit and W. Wiersinga, "European Consensus for the Management of Patients with Differentiated Thyroid Carcinoma of the Follicular Epithelium," European Journal Endocrinology, Vol. 154, No. 6, 2006, pp. 787-803. doi:10.1530/eje.1.02158

[16] O. Gimm, F. W. Rath and H. Drall, "Pattern of Lymph Node Metastases in Papillary Thyroid Carcinoma," British Journal of Surgery, Vol. 85, No. 2, 1998, pp. 252-254. doi:10.1046/j.1365-2168.1998.00510.x

[17] O. Ozaki, K. Ito, K. Kobayash, A. Suzuki and Y. Manabe, "Modified Neck Dissection for Patients with Nonadvanced, Differentiated Carcinoma of the Thyroid," World Journal of Surgery, Vol. 12, 1988, pp. 825-829. doi:10.1007/BF01655487

[18] Y. Ito and A. Miyauchi, "Lateral and Mediastinal Lymph Node Dissection in Differentiated Thyroid Carcinoma: Indications, Benefits, and Risks," World Journal of Surgery, Vol. 31, No. 5, 2007, pp. 905-915. doi:10.1007/s00268-006-0722-0

[19] M. E. Kupferman, Y. E. Weinstock, A. A. Santillan, A. Mishra, D. Roberts, G. L. Clayman and R. S. Weber, "Predictors of Level V Metastasis in Well-Differentiated Thyroid Cancer," Head Neck, Vol. 30, No. 11, 2008, pp. 1469-1474. doi:10.1002/hed.20904

[20] I. Schweizer, P. U. Heitz, E. Gemsenjager, A. Perren, B. Seifert and G. Schu, "Lymph Node Surgery in Papillary Thyroid Carcinoma," Journal of the American College of Surgeons, Vol. 197, No. 2, 2003, pp. 182-190. doi:10.1016/S1072-7515(03)00421-6
[21] M. R. Pelizzo, D. Rubello, I. M. Boschin, A. Piotto, C. Paggetta, A. Toniato, G. L. De Salvo, A. Giuliano, G. Mariani and D. Casara, "Contribution of SLN Investigation with $99 \mathrm{mTc}-$ Nanocolloid in Clinical Staging of Thyroid Cancer: Technical Feasibility," European Journal of Nuclear Medicine and Molecular Imaging, Vol. 34, No. 6, 2007, pp. 934-938.

doi:10.1007/s00259-006-0316-y

[22] R. Dzodic, I. Markovic, M. Inic, N. Jokic, I. Djurisic, M. Zegarac, G. Pupic, Milovanovic Z, V. Jovic and N. Jovanovic, "Sentinel Lymph Node Biopsy May Be Used to Support the Decision to Perform Modified Radical Neck Dissection in Differentiated Thyroid Carcinoma," World Journal of Surgery, Vol. 30, No. 5, 2006, pp. 841-846. doi:10.1007/s00268-005-0298-0

[23] J. L. Roh and C. I. Park, "Sentinel Lymph Node Biopsy as Guidance for Central Neck Dissection in Patients with Papillary Thyroid Carcinoma," Cancer, Vol. 113, No. 7, 2008, pp. 1527-1531. doi:10.1002/cncr.23779

[24] A. Machens, S. Hauptmann and H. Dralle, "Lymph Node Dissection in the Lateral Neck for Completion in Central Node-Positive Papillary Thyroid Cancer," Surgery, Vol. 145, No. 2, 2009, pp. 176-181. doi:10.1016/j.surg.2008.09.003

[25] M. Ducci, M. Appetecchia and M. Marzetti, "Neck dissection for Surgical Treatment of Lymphnode Metastasis in Papillary Thyroid Carcinoma," Journal of Experimental \& Clinical Cancer Research, Vol. 16, No. 3, 1997, pp. 333-335.

[26] A. P. Coatesworth and K. MacLennan, "Cervical Metastasis in Papillary Carcinoma of the Thyroid: A Histopathological Study," International Journal of Clinical Practice, Vol. 56, No. 4, 2002, pp. 241-242.

[27] Y. Ito, C. Tomoda, T. Uruno, Y. Takamura, A. Miya, K. Kobayashi, F. Matsuzuka, K. Kuma and A. Miyauchi, "Papillary Microcarcinoma of the Thyroid: How Should It Be Treated?" World Journal of Surgery, Vol. 28, No. 11, 2004, pp. 1115-1121. doi:10.1007/s00268-004-7644-5 